\title{
Identification of the noise using mathematical modelling
}

\author{
Josef Dobeš $^{1, a}$, Milada Kozubková ${ }^{1}$ and Miroslav Mahdal ${ }^{2}$ \\ ${ }^{1}$ Department of Hydromechanics and hydraulic equipment, Technical University of Ostrava, Ostrava, Czech Republic \\ ${ }^{2}$ Department of Control Systems and Instrumentation, Technical University of Ostrava, Ostrava, Czech Republic
}

\begin{abstract}
In engineering applications the noisiness of a component or the whole device is a common problem. Currently, a lot of effort is put to eliminate noise of the already produced devices, to prevent generation of acoustic waves during the design of new components, or to specify the operating problems based on noisiness change. The experimental method and the mathematical modelling method belong to these identification methods. With the power of today's computers the ability to identify the sources of the noise on the mathematical modelling level is a very appreciated tool for engineers. For example, the noise itself may be generated by the vibration of the solid object, combustion, shock, fluid flow around an object or cavitation at the fluid flow in an object. For the given task generating the noise using fluid flow on the selected geometry and propagation of the acoustic waves and their subsequent identification are solved and evaluated. In this paper the principle of measurement of variables describing the fluid flow field and acoustic field are described. For the solution of fluid flow a mathematical model implemented into the CFD code is used. The mathematical modelling evaluation of the flow field is compared to the experimental data.
\end{abstract}

\section{The Possibilities of the Acoustic Task Solution}

The numerical solutions of the generation and propagation of the acoustic waves help reduce the noise level of the individual components or the equipment altogether. Today the possibility of numerical modelling of the generation and propagation of acoustic waves is a very appreciated tool. These numerical simulations are compared with the data obtained by experimental measurements, which is, particularly for measurements of noise or sound intensity, highly expensive procedure as regards the experimental equipment and staff.

Noise can be generated by several different methods that, from physical perspective, can be divided into the following groups [1]:

- noise generated by the friction of solid parts gearboxes,

- noise generated by the vibration of the components engine covers,

- noise generated by the combustion - piston engines,

- noise generated by the shocks - explosions, cavitation,

- noise generated by the vortex shedding structures aerodynamic noise.

For the numerical modelling of the fluid flow using CFD (Computational Fluid Dynamics) code and the subsequent modelling of propagation of acoustic waves the most interesting applications are in the field of cavitation behaviour of the fluid elements and in the field of the flown-around obstacles, known as aeroacoustic noise (CAA - Computational aeroacoustics).

\section{The numerical solution of the aeroacoustic task}

From the viewpoint of the numerical solution of the generation and the propagation of the acoustic waves through the fluid flow (cavitation, aeroacoustic noise) is the solution of the problem split into two particular parts, each of them is solved separately in the given order.

In the first part the problem of the description of the flow field of flowing fluid is solved through the NavierStokes equations, the continuity equation and additional equations for the appropriate turbulence model are numerically solved.

The second part of the task lies in dealing with the propagation of acoustic waves generated by the solved flow field in the given environment and their subsequent evaluation. On the simple task of the flown-around cylinder the method how to solve aeroacoustic task is shown. The cylinder is solved as a planar 2D task. The measuring section of the wind tunnel and inserted real flown-around object is the geometry.

The experimental work was focused on the measurement of the velocity profiles for the cylinder and the measurement of the sound intensity. Due to large complexity of the calculations and the experimental work

\footnotetext{
$\overline{{ }^{a} \text { Corresponding author: josef.dobes } @ v s b . c z ~}$
} 
is the flown-around cylinder solved at one value of the velocity $\mathbf{u}$ and thus Reynolds number $R e$.

\subsection{CFD tool OpenFOAM}

OpenFOAM is a tool for solving CFD simulation for a wide range of engineering applications, which is based on the programming language $\mathrm{C} / \mathrm{C}++$. In the basic configuration it contains over 80 solvers and 170 applications enabling pre- and post-processing [2]. The numerical task is defined using the following directory structure. The main directory named "20_ms" has the following subdirectories:

- „system“ - solver setup in the file "controlDict", tolerances and numerical schemes for solving of the partial differential equations,

- „constant“ - contains information about the computational mesh, the physical properties of the flowing medium and the selected turbulent model,

- „0“ - time directory contains information about boundary and initial conditions for solved tasks.

For this task the version of program OpenFOAM 2.3. $\mathrm{x}$ is applied. It is an air flow at a velocity $\mathbf{u}=20 \mathrm{~m} \mathrm{~s}^{-1}$ of unaffected field, we consider the flow of the liquid air - incompressible flow and as the numerical solver pisoFoam is selected. The Reynolds number for the cylinder at diameter $d=20 \mathrm{~mm}$, at velocity $\mathbf{u}=20 \mathrm{~m} \mathrm{~s}^{-1}$ is $R e=26042$.

\subsection{The creation of geometry and mesh}

The geometry and the computational mesh was created in OpenFOAM software environment using the appropriate commands, checked in a graphical environment Paraview and subsequently modified or approved and then it went through the numerical calculation.

The geometry and mesh for this task is created directly by "blockMesh" command. The appropriate command creates geometry and mesh in a few steps:

- creates points placed in a 3D coordinate system,

- links these points into blocks,

- divides the blocks into individual cells according to parameters specified by the user,

- sets boundary conditions of the task.

OpenFOAM allows creating only a 3D object. For numerical solution of a 2D task you need to specify a special boundary condition - "empty".

\subsection{The numerical solution of the fluid flow - $k$ - epsilon turbulence Standard model}

The partial differential equations describing fluid flow are defined for time averaging values, while in the equations Einstein summation rule is used. The continuity equation for incompressible fluid has the form $[2,3]$ :

$$
\frac{\partial u_{\mathrm{j}}}{\partial x_{\mathrm{j}}}=0
$$

where the member $\mathbf{u}_{\mathrm{j}}$ is $j$-th component of velocity, the index $\mathrm{j}$ indicates the counting index.
For incompressible fluid the Navier - Stokes equation can be written as:

$$
\frac{\mathrm{D} u_{\mathrm{i}}}{\mathrm{D} t}=-\frac{1}{\rho} \frac{\partial p}{\partial x_{\mathrm{i}}}+v \frac{\partial^{2} u_{\mathrm{i}}}{\partial x_{\mathrm{j}}{ }^{2}}+f_{\mathrm{i}}
$$

where the member $\frac{\mathrm{D}}{\mathrm{D} t}$ indicates substantial derivative, which is a kind of total derivatives following the movement. The member $v$ is the kinematic viscosity, $\mathbf{u}_{\mathrm{i}}$ is $i$-th component of velocity vector, $p$ denotes pressure, $\rho$ density of fluid and, generally, the member $f_{\mathrm{i}}$ symbolizes $i$-th component of the external volume forces (gravitational, centrifugal force). The used turbulent kepsilon Standard model is a two-equation turbulent model that adds next two transport equations to solving the equations mentioned above. One of them is the equation for turbulent kinetic energy $k$ :

$$
\begin{aligned}
& \frac{\partial}{\partial t}(\rho \cdot k)+\nabla \cdot(\rho k \vec{u})=\nabla\left(\left(\mu+\frac{\mu_{t}}{\sigma_{k}}\right) \nabla k\right)+ \\
& G_{k}+G_{b}-\rho \cdot \varepsilon-Y_{M}+S_{k}
\end{aligned}
$$

the second one is the equation for the dissipation rate $\varepsilon$ :

$$
\begin{aligned}
& \frac{\partial}{\partial t}(\rho \cdot \varepsilon)+\nabla \cdot(\rho \varepsilon \vec{u})=\nabla\left(\left(\mu+\frac{\mu_{t}}{\sigma_{\varepsilon}}\right) \nabla \varepsilon\right)+ \\
& C_{1 \varepsilon} \frac{\varepsilon}{k}\left(G_{k}+C_{3 \varepsilon} \cdot G_{b}\right)-C_{2 \varepsilon} \rho \cdot \frac{\varepsilon^{2}}{k}+S_{\varepsilon}
\end{aligned}
$$

In the literature we can find a detailed description of this model [3]. Such modified differential equations are solved by finite volume method in program OpenFOAM.

\subsection{Boundary conditions}

The boundary conditions for the flown-around cylinder are specified in the files in the " 0 " directory. At the inlet into the geometry the velocity $\mathbf{u}$ and the turbulence quantities $k$ and $\varepsilon$ were assigned. The pressure $p=0 \mathrm{~Pa}$ was assigned at the outlet as relative. The gravity is set by the file , $\mathrm{g}^{\text {" }}$ in the ,constant" directory.

\subsection{The numerical solution of acoustic waves}

From the viewpoint of the possible solutions the aeroacoustic task the access using numerical methods can be grouped as follows [4]:

- a method of direct simulation of noise (CAA) - this method is the most demanding in terms of time step size and cell size of the mesh. Small fluctuations of pressure $p$ and velocity $\mathbf{u}$ are solved directly which carries information of the noise generated by the flow. The noise source and the receiver must be placed in the solved area. - a method when the solution of CFD simulation serves to define the noise sources for specialized acoustic programs - the acoustic waves propagation is solved.

- a method of modelling by the acoustic analogy - CFD simulation serves to calculate source members. In the selected parts of the calculation area, where the noise generation is anticipated, pressure $p$, velocity $\mathbf{u}$ and for compressible media the density $\rho$ in each time step are recorded. From these records the propagation of acoustic waves in a "stationary" fluid is calculated. 


\subsubsection{The acoustic analogy}

The acoustic analogy is used to describe the connection between the flow field and the acoustic field emerging due to flowing. It describes the relationship between the propagation of acoustic waves and its causes (sources). The acoustic analogy can be divided to acoustic analogy based on the fluid density $\rho$, acoustic analogy based on a logarithmic ratio of the pressures in the fluid, and acoustic analogy based on the enthalpy of the fluid $H$ [5]. The Curle's analogy falls into analogy derived and subsequently expressed depending on the density of the fluid $\rho$. This is a special version of analogy Ffowcs Williams-Hawkings (abbreviated FW-H) that is derived from the basic Lighthill's analogy. The theory and the derivation of this analogy can be found in literatures [5]. If the surface $S$ in the FW-H equation is assumed to be rigid and it does not move, the equivalent monopole surface source distribution $q_{\mathrm{ws}}$ and its substitute volume dipole and quadrupole source distributions $f_{\mathrm{WVc}}$ and $T_{\mathrm{WVc}}$ disperse as well as the corresponding density perturbation components $\rho_{\nu S}^{\prime}, \rho_{\mathrm{fVc}}^{\prime}$ and $\rho_{\mathrm{TVc}}^{\prime}$ [5]. This leads to Curle's equation:

$$
\begin{aligned}
& \frac{\partial^{2} \rho^{\prime}}{\partial t^{2}}-c^{2} \nabla^{2} \rho^{\prime}=-\nabla\left[\vec{f}_{W S} \delta\left(w_{1}-w_{10}\right)\right] \\
& +\nabla \nabla:\left[\overline{\bar{T}}_{L} H\left(w_{1}-w_{10}\right)\right]
\end{aligned}
$$

The detailed description of the individual variables can be found in the appropriate literature sources $[1,5]$.

\subsubsection{The implementation of Curle's analogy}

The Curle's analogy implementation was done according to literature [6] for the version of program OpenFOAM 2.3.x. The implementation itself is composed of several consecutive steps that guarantee the proper function of individual libraries for evaluation of acoustic quantities using Curle's acoustic analogy. It is necessary to implement a dynamic library to obtain the variables for the possibility of calculating the acoustic pressure, the library of analogy for the calculation of acoustic quantities and the library for the data evaluation.

\subsubsection{The setup of Curle's acoustic analogy}

The setup is done in the file named "CurleAnalogy“ in the directory ,system“. The start and end time of sensing acoustic quantities, the source object - cylinder, the speed of sound in the medium c, the reference density $\rho$, pressure $p$ and the reference sound pressure $p_{A}$ are defined in this file. Furthermore, receivers' (the probes) positioning, the sampling frequency for evaluating a fast Fourier transform are defined. More information about this analogy can be obtained from the literature [6].

\subsubsection{The results of aeroacoustic simulation using by program OpenFOAM}

In the introduction of the Chapter 2 aeroacoustic task solution is generally described. In this section, the evaluation of numerical simulation task is indicated, more precisely the acoustic part. The aerodynamic part of the task is evaluated and also compared with the experimental data in Chapter 4.1.

The comparison of the numerical simulation with the experimental measurements for acoustic part has not been carried out yet. The experimental acoustic work is in the phase of research and development for the most accurate and appropriate measurement method, which will deal appropriately with the issue of the aeroacoustic task and verify the numerical simulations properly.

The result of the acoustic task's numerical simulation is plotting of sound pressure level depending on the frequency of individual receivers R1 until R5, which were located according to the drawing below (all dimensions in the drawings in this paper are in $\mathrm{mm}$ ).

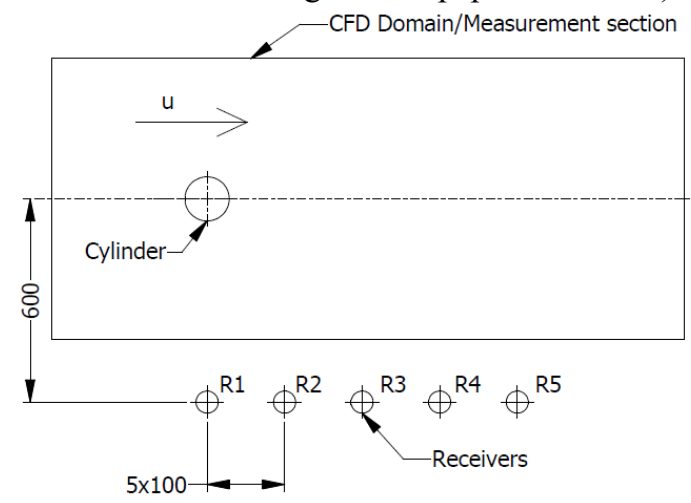

Figure 1. The drawing of receivers' location in acoustic simulation

The aeroacoustic simulation result is shown in the following chart. From the graphical evaluation it can be seen that the first significant peak of the sound pressure level (abbreviated SPL) is at the frequency $143 \mathrm{~Hz}$. This value is lower than vortex shedding frequency of the flown-around cylinder. This value is recorded for all the receivers, when the values vary in the size of SPL where with increasing distance from the cylinder the SPL decreases. The highest sound pressure level at this frequency is $123 d B$ (for receiver R1), which is a very high value. The second significant peak is at the frequency $285 \mathrm{~Hz}$. This value is approximately double compared to the first significant frequency. The highest value of SPL at this frequency is $80 d B$. The results are not processed using any weighting filter (A-weighted or C-weighted).

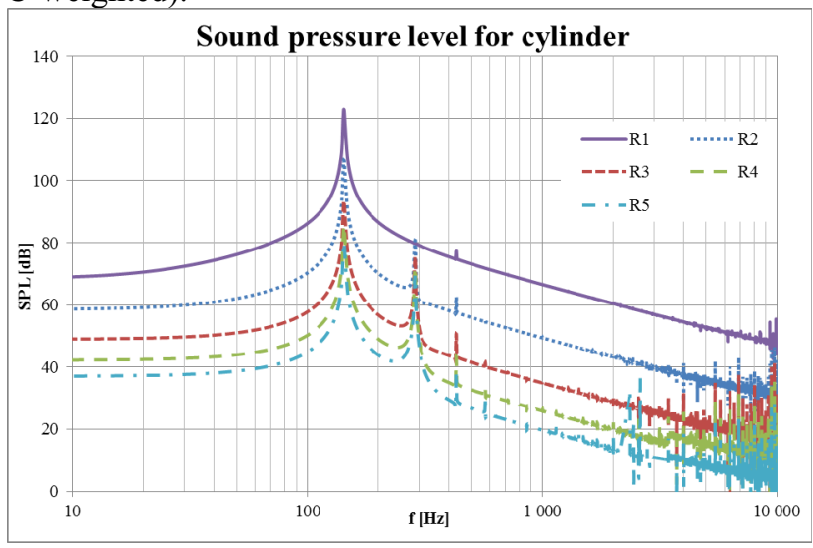

Figure 2. The evaluation SPL for acoustic receivers 


\section{The experimental part of acoustic task}

The experimental part of task was performed using a wind tunnel on the Department Hydromechanics and hydraulic equipment of Technical university of Ostrava.

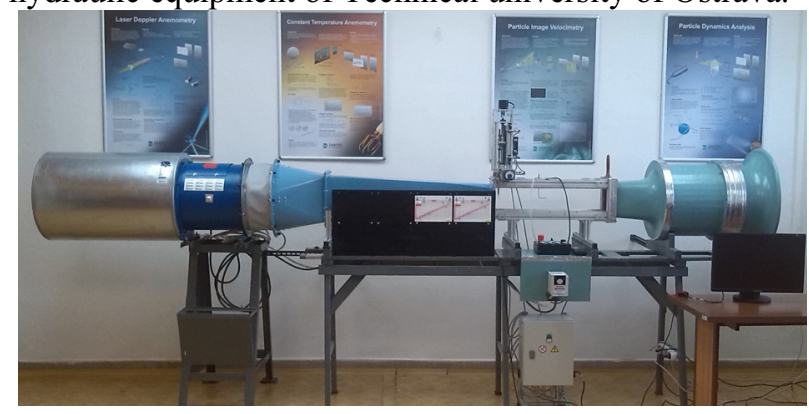

Figure 3. The experimental device for measuring task

In the first part the measurement was carried out to verify the numerically calculated flow field with the experimental data obtained with the hot-wire probe MiniCTA. In the second part, to obtain the data describing the acoustic field, the experimental work was performed by measuring the wind tunnel without obstacle by the intensity probe.

\subsection{The measurement of the flow field}

The measurement of velocity profiles in a defined distance behind the flown-around cylinder was done by the one-wire probe MiniCTA type 55P14. The probe was clamped into traversing device to ensure the most accurate position for measurement of velocity component in the flow direction.

The velocity profiles for the cylinder were measured in the distances: $2 \times D, 3 \times D, 4 \times D, 5 \times D, 6 \times D$ a $7 \times D$, when $D$ is the cylinder diameter $(20 \mathrm{~mm})$. In these distances the velocity profile perpendicular to the main direction of the velocity vector was measured.

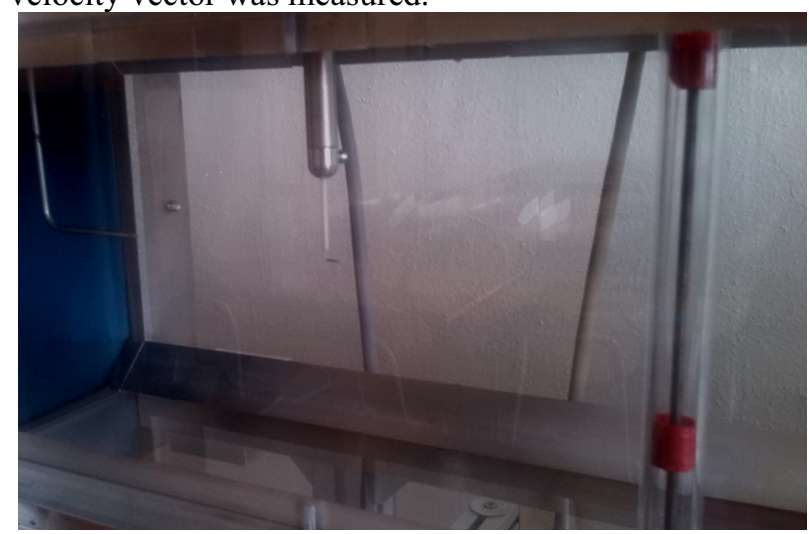

Figure 4. The detail of measuring velocity profile for cylinder

The velocity profiles for cylinder were obtained by measuring at 14 points in a straight line which is perpendicular to the direction of the velocity vector. The distance between two consecutive points was set at $6 \mathrm{~mm}$. A finer measurement, the smaller the distance between two consecutive points, was rejected because of limited capacity of the disk storage. The drawing of the velocity profiles measurement is shown in the Figure 5.

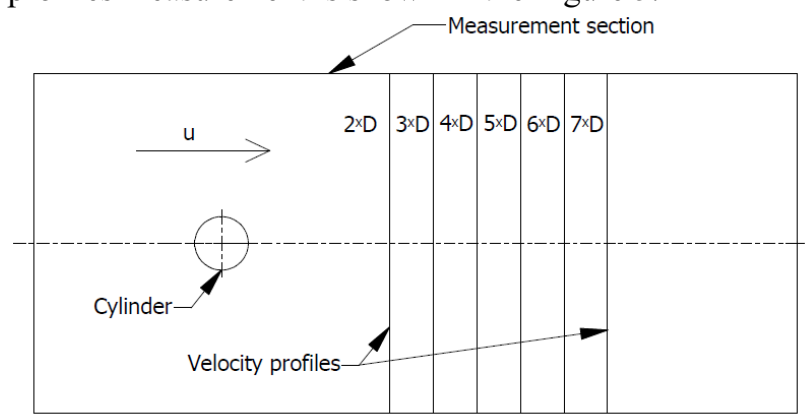

Figure 5. The drawing of velocity profiles measurement

Subsequently, all experimentally obtained velocity profiles were compared with the results of numerical simulation for checking of numerical CFD code setup.

Fast Fourier transform of the measured velocity data to determine the vortex shedding frequency $f$ and the calculation of experimental Strouhal number $S t$ was also done. This value was compared to the results of numerical simulation and literature in the Table 1.

\subsection{The measurement of the acoustic field}

For the acoustic measurements so-called Intensity probes are suitable measuring instruments. These are based on the measurement of sound pressure in two nearby points, these probes are often termed as $p-p$ probes or twomicrophone probes. For the measurement the high quality condenser microphones are used and the most common setup of those is face to face. The distance between the microphones is defined using spacers.

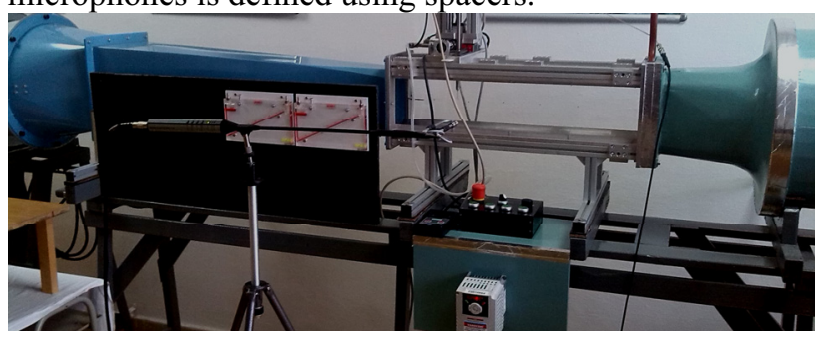

Figure 6. The intensity probe for acoustic measurement

Subsequently, the measurement was evaluated using the relevant analyser and software. As intensity probe the probe type 3599 from the company Bruel \& Kjaer was used. The drawing of the measurement is shown in the Figure 7. There are also shown the measuring points (A0 - A4) with the respective designation of these points.

\section{The comparison of numerical and experimental data}

In this part of the paper a verification of experimental data with data obtained by numerical simulation is done. In the first part the experimentally obtained velocity profiles were compared with numerically obtained velocity profiles and the numerically obtained drag coefficient $C_{\mathrm{d}}$ and Strouhal number $S t$ were compared 
with literature. In section 4.2 the results obtained by the intensity probe measuring are presented. For better clarity, this section is divided into comparison of the aerodynamic part - velocity profiles, drag coefficient $C_{\mathrm{d}}$ and Strouhal number St. For the acoustic part of the task measuring the tunnel without cylinder is done. To obtain data describing the acoustic behaviour of whole system, the acoustic measurement serves as a reference for the future measuring with obstacle

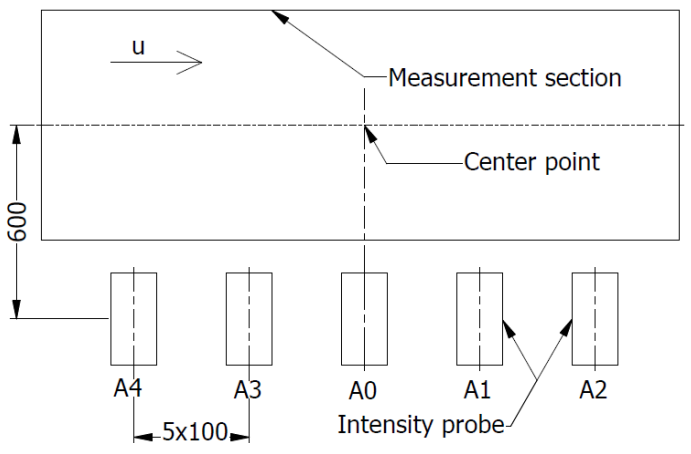

Figure 7. The acoustical measurement drawing for tunnel

\subsection{The aerodynamic experimental part}

To compare experimental and numerical data averaging velocity profiles for the flown-around cylinder were selected. As a complement, a comparison of the drag coefficient $C_{\mathrm{d}}$ and Strouhal number $S t$ with literature for cylinder is done [7]. In the Figure 8 experimentally obtained velocity profiles for the cylinder are shown.

In the graphic representation, the $\mathrm{X}$-axis shows the measuring points, when point $\mathrm{X} 8$ is a measurement of the velocity profile past the cylinder axis. The $\mathrm{Y}$-axis shows the arithmetic average of measured velocity. For the comparison the experimental data with the numerical results profiles $2 \times D$ and $3 \times D$ were selected. Parameters of the measurement were sampling frequency $f=480 \mathrm{~Hz}$ which corresponds to vortex shedding frequency of cylinder multiplied by 2.5 for compliance with the Nyquist- Kotelnikov theorem. The number of samples for all measurement was 4096.

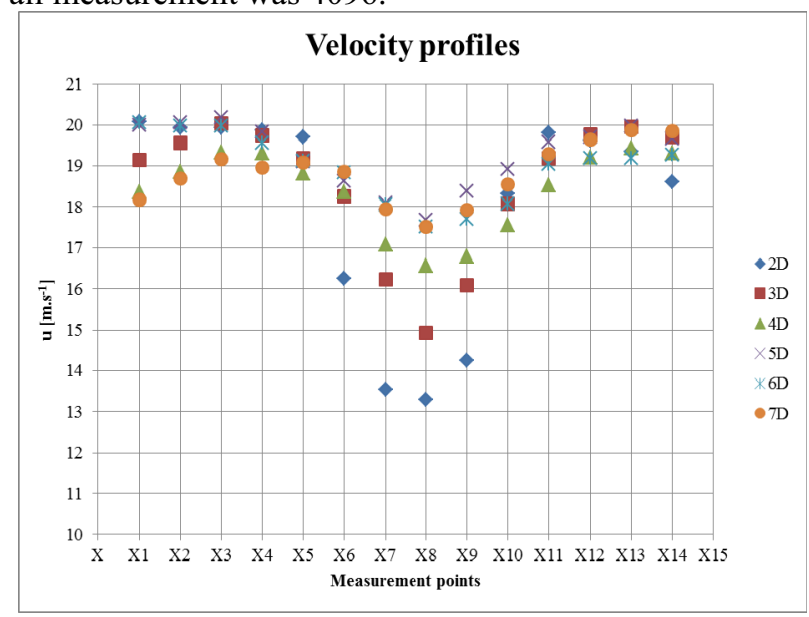

Figure 8. The experimental velocity profiles - cylinder

\subsubsection{The comparison of velocity profiles for cylinder}

The following chart shows comparing the velocity profiles between the numerical simulations indicated in the graph as "OpenFOAM 2D" and "OpenFOAM 3D", with experimental data, indicated in the graph as the "2D" and "3D" for the cylinder.

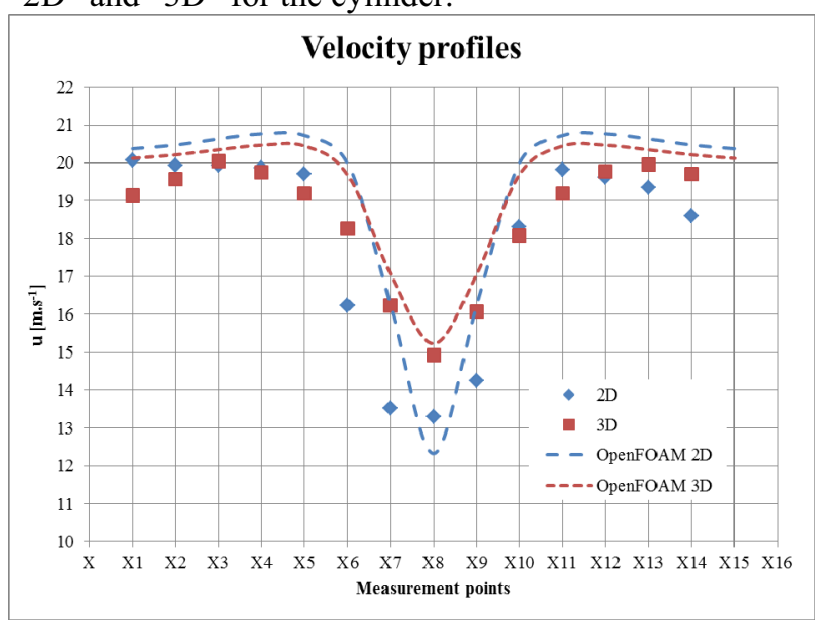

Figure 9. The velocity profiles comparison for cylinder

The comparison shows that the velocity profiles are identical in the field behind the flown-around obstacle. In the direction from the cylinder to the wall there are already differences which may be caused by short measuring record and by high sensitivity of the hot-wire probe on the turbulent fluctuations. These fluctuations arise already at the inlet to the measuring section of the tunnel and are not considered for the numerical simulation. To reduce this error in the future, for numerical simulation, the experimentally obtained velocity profile will be entered at the inlet to the measuring section. The technical solution by upgrading the suction side is also being prepared. The newer section should contain a suction sieve, together with the stabilizing honeycomb for calming flowing, and thus achieve better velocity profile of the measuring section.

\subsubsection{The drag coefficient, Strouhal number}

To calculate the drag coefficient $C_{\mathrm{d}}$ the library „libforces.so“ can be used. It uses the file „forceCoeffs“, in directory „system“. It allows calculating the drag forces (pressure, viscous) and the coefficients on the relevant, user defined, flown-around obstacle. Furthermore, in this file velocity $\mathbf{u}$, density $\rho$, reference length $L$ and cross sectional area $S$ to perpendicular to the flow direction are defined. The drag coefficient $C_{\mathrm{d}}$ can be computed by the equation:

$$
C_{\mathrm{d}}=\frac{2 \cdot F_{\mathrm{d}}}{\rho \cdot u^{2} \cdot S}
$$

where $F_{\mathrm{d}}$ indicates total drag force which consists of a viscous and pressure drag forces, $\rho$ indicates the density of fluid, $\mathbf{u}$ indicates velocity in unaffected field, $S$ represents cross sectional area perpendicular to the velocity vector $\mathbf{u}$. 
The evaluation of Strouhal number $S t$ was carried out by calculating according to the following equation:

$$
S t=\frac{f \cdot l}{u}
$$

where $f$ indicates vortex shedding frequency, $l$ represents the characteristic dimension (diameter of cylinder) and $\mathbf{u}$ represents the velocity. The frequency $f$ was calculated using the inverse value of the difference between the two closest points of equal value of velocity $\mathbf{u}$ acquired from the graphical representation of $t / \mathbf{u}$ dependence. In the following table the comparison of numerically acquired data with data from the literature/experiment is done.

Table 1. The comparison of aerodynamic variables for cylinder

\begin{tabular}{|c|c|c|c|}
\hline \multirow{2}{*}{ Parameter } & \multicolumn{3}{|c|}{ Cylinder Re $=26042$} \\
\cline { 2 - 4 } & Literature & $\begin{array}{c}\text { Numerical } \\
\text { Results }\end{array}$ & Experiment \\
\hline $\begin{array}{c}\text { Drag } \\
\text { coefficient }\end{array}$ & 1.009 & 1.066 & - \\
\hline $\begin{array}{c}\text { Strouhal } \\
\text { number }\end{array}$ & 0.192 & 0.189 & 0.179 \\
\hline
\end{tabular}

\subsection{The acoustic experimental part of the task}

As an example measurement evaluation for wind tunnel without inserted obstacles is shown. This measurement is used as a reference for measurement evaluation of acoustic variables for arbitrary obstacle.

The sampling frequency was set on the value 32.7 $k H z$, the length of the record was $10 \mathrm{~s}$. The possibility of automatic averaging of measured data using by intensity probe software was used. Positive values were determined by the intensity sound in the direction from the measuring section of the wind tunnel, the negative intensity sound values were determined from the opposite side (room), where the measurement was carried out. This determination of positive/negative values was evaluated by a simple experiment - clap hands between the probe and the measuring section of the tunnel (for positive values), or behind the probe (for negative values) and then record this phenomenon and evaluate.

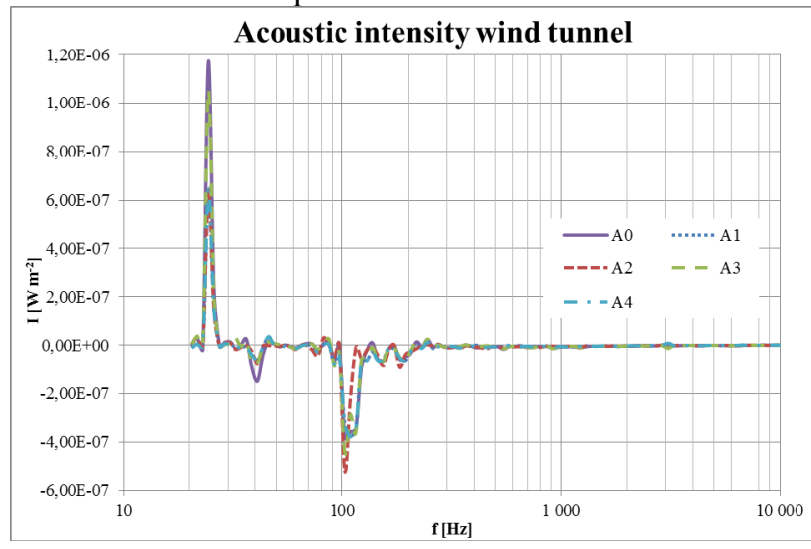

Figure 10. The evaluation of the intensity of the sound

At every point A0 - A4 three measurements were carried out. Subsequently, the average value of these three measurements was calculated and used to plot into the graph in the Figure 10.
The first significant (positive) peak is at the frequency of $25 \mathrm{~Hz}$, which corresponds to the configuration of the frequency inverter. The value for the inverter was set to $24.8 \mathrm{~Hz}$. The second significant (negative) peak is observed at the frequency of $109 \mathrm{~Hz}$ which can be attributed to the actual velocity of the 6-bladed fan. The silencer which is connected behind the fan causes the frequencies are reduced.

\section{The final evaluation of task}

In this paper a numerical solution of the noise generated by the cylinder was solved. As a CFD tool OpenFOAM was used. The turbulent model k-epsilon model Standard was selected. The measurement of velocity profiles using the hot-wire anemometer was done. Furthermore, measurement by the intensity probe in the tunnel without flown-around obstacle was carried out.

The comparison between the numerical methods and experiment was performed for velocity profiles. The comparison of velocity profiles is made in the Figure 9 for the cylinder. The comparison of the values (drag coefficient $C_{d}$, Strouhal number $S t$ ) is provided in the Table 1. The comparison of numerically obtained drag coefficient and Strouhal number with the literature data indicates the values are in good compliance. In the second part of the task, after solving the aerodynamic task; the numerical solving of the acoustic wave propagation was done. To solve this problem the Curle's analogy through the implementation in the source code of the program OpenFOAM was used. The acoustic simulation results are shown in the Figure 2. The experimental part was carried out using the intensity probe. Significant peaks at two frequencies are presented.

The solving of the aeroacoustics issue will continue. In the numerical calculation area the solution of the acoustic pressure signal transition through a layer of plexiglass needs to be found. It is the border between the measuring section and the outside environment, which significantly influences the passing signal. In the experimental area the optimization of experiments will be made to reduce the noise, which is not the investigation subject and degrades the measurement for our needs.

\section{Acknowledgement}

This paper was funded by specific research on the Technical University of Ostrava named "Zkoumání dynamiky tekutinových systému”, code SP2015/95.

\section{References}

1. Wagner, C. Large-Eddy Simuation for Acoustics. 470 (Cambridge University Press, 2012)

2. OpenFOAM The Open Source CFD Toolbox User Guide, Version 2.3.1. 221 (2014)

3. Ansys Fluent Theory Guide, Release 15.0. Ansys, Inc., 814 (2013)

4. Verhoeven, Olivier. Trailing Edge Noise Simulations using IDDES in OpenFOAM. 139 (Delft, 2011) 
5. Uosukainen, S. Foundations of acoustic analogies [Akustisten analogioiden perusteet]. 103 (VTT Technical Research Centre of Finland, 2011)

6. Kraposhin, M. V. Strizhak, S. V. How to implement simple acoustic analogy in OpenFOAM. 49 (8th OpenFOAM Workshop, Jeju, Korea 2013)

7. Roshko, A. Experiments on the flow past a circular cylinder at very high Reynolds number. 12 (Journal of Fluid Mechanics. 1961)

8. Ayachit, U. The ParaView Guide, 239 (2015) 